\title{
In vitro and in vivo quantification of chloroprocaine release from an implantable device in a piglet postoperative pain model
}

This article was published in the following Dove Press journal: Journal of Pain Research

\author{
Simona De Gregori' \\ Manuela De Gregori ${ }^{1-4}$ \\ Nora Bloise $e^{5,6}$ \\ Dario Bugada $3,4,7$ \\ Mariadelfina Molinaro' \\ Claudia Filisetti ${ }^{8}$ \\ Massimo Allegri ${ }^{3,9}$ \\ Michael E Schatman ${ }^{3,10,11}$ \\ Lorenzo Cobianchi ${ }^{12,13}$ \\ 'Clinical and Experimental \\ Pharmacokinetics Unit, Fondazione IRCCS \\ Policlinico San Matteo, Pavia, Italy; ${ }^{2}$ Pain \\ Therapy Service, Fondazione IRCCS \\ Policlinico San Matteo, Pavia, Italy; ${ }^{3}$ Study \\ in Multidisciplinary Pain Research Group, \\ Parma, Italy; ${ }^{4}$ Young Against Pain Group, \\ Parma, Italy; ${ }^{5}$ Department of Molecular \\ Medicine, Centre for Health Technologies, \\ INSTM UdR of Pavia, University of Pavia, \\ Pavia, Italy; 'Department of Occupational \\ Medicine, Toxicology and Environmental \\ Risks, Istituti Clinici Scientifici Maugeri, \\ IRCCS, Lab of Nanotechnology, Pavia, \\ Italy; ${ }^{7}$ Emergency and Intensive Care \\ Department - ASST Papa Giovanni XXIII, \\ Bergamo, Italy; " "V. Buzzi" Children \\ Hospital, Pediatric Surgery, Milan, Italy; \\ ${ }^{9}$ Anesthesia and Intensive Care Service, \\ IRCCS MultiMedica Hospital, Sesto San \\ Giovanni, Milano, Italy; ${ }^{10}$ Research and \\ Network Development, Boston Pain Care, \\ Waltham, MA, USA; "'Department of Public \\ Health and Community Medicine, Tufts \\ University School of Medicine, Boston, \\ MA, USA; ${ }^{2}$ General Surgery Department, \\ Fondazione IRCCS Policlinico San Matteo, \\ Pavia, Italy; ${ }^{13}$ Department of Clinical, \\ Surgical, Diagnostic and Pediatric Sciences, \\ University of Pavia, Pavia, Italy
}

Correspondence: Simona De Gregori

Clinical and Experimental Pharmacokinetics

Unit, Fondazione IRCCS Policlinico San Matteo,

Viale Golgi 19, 27I00 Pavia, Italy

$\mathrm{Tel}+39382503647$

Fax +39382503618

Email simo.degregori@gmail.com
Background: The pharmacokinetic properties and clinical advantages of the local anesthetic chloroprocaine are well known. Here, we studied the pharmacokinetic profile of a new hydrogel device loaded with chloroprocaine to investigate the potential advantages of this new strategy for postoperative pain (POP) relief.

Materials and methods: We performed both in vitro and in vivo analyses by considering plasma samples of four piglets receiving slow-release chloroprocaine. To quantify chloroprocaine and its inactive metabolite 4-amino-2-chlorobenzoic acid (ACBA), a HPLC-tandem mass spectrometry (HPLC-MS/MS) analytical method was used. Serial blood samples were collected over 108 hours, according to the exposure time to the device.

Results: Chloroprocaine was consistently found to be below the lower limit of quantification, even though a well-defined peak was observed in every chromatogram at an unexpected retention time. Concerning ACBA, we found detectable plasma concentrations between $\mathrm{T}_{0}$ and $\mathrm{T}_{12 \mathrm{~h}}$, with a maximum plasma concentration $\left(\mathrm{C}_{\max }\right)$ observed 3 hours after the device application. In the in vitro analyses, the nanogel remained in contact with plasma at $37^{\circ} \mathrm{C}$ for 90 minutes, 3 hours, 1 day, and 7 days. Chloroprocaine $\mathrm{C}_{\max }$ was identified 1 day following exposure and $\mathrm{C}_{\min }$ after 7 days, respectively. Additionally, ACBA reached the $\mathrm{C}_{\max }$ following 7 days of exposure. Conclusion: A thorough review of the literature indicates that this is the first study analyzing both in vivo and in vitro pharmacokinetic profiles of a chloroprocaine hydrogel device and is considered as a pilot study on the feasibility of including this approach to the management of POP. Keywords: postoperative outcome, hydrogel device, chloroprocaine, ACBA, pharmacokinetics

\section{Introduction}

The mechanism of local anesthetics (LAs) in blocking the transmission of painful stimuli to the brain is well known. ${ }^{1}$ Chloroprocaine, an ester-type LA, is less cardiotoxic than most others, but it is characterized by an extremely short half-life. ${ }^{2}$ New delivery systems based on hydrogel devices may effectively and safely extend the LA's analgesic action.,

Thanks to their unique properties, including biocompatibility, flexible methods of synthesis, network structure, and stability of the incorporated bioactive molecules, hydrogel devices have sparked particular interest in use in drug delivery applications. ${ }^{5}$ Currently, a wide number of natural polymers (ie, chitosan, pectin, and alginate) are employed for hydrogel preparation due to their capability of mimicking biological structures and the fact that they are well tolerated inside the human body.,6

Postoperative pain (POP) remains a challenge. ${ }^{7} \mathrm{POP}$ is a common feature in all surgical procedures, and it can have a profound impact on patients' outcomes. LAs represent a cornerstone for effective postoperative analgesia ${ }^{8}$ because they block 
nociceptive stimulus from the periphery and provide pain relief both at rest and during movement. However, LAs have limited duration of action, and even with modern long-acting formulations, analgesic efficacy is limited to the first day following surgery. Unfortunately, POP continues much longer than the first day, and as soon as the effect of the analgesic block decreases, nociceptive and hyperalgesic mechanisms can start again. ${ }^{9}$ Since prolonged uncontrolled pain in the perioperative period can lead to immediate and long-term complications (including pain chronicization), there is strong interest in prolonging nociceptive blockage. Catheters are used to provide continuous infusion of LAs but, despite their efficacy, they require additional efforts for management by care providers and patients, and complications can occur. ${ }^{10}$ New strategies for prolonged and controlled release of LAs are required, together with the use of dedicated LAs with optimal pharmacokinetic profiles for continuous infusion (ie, low toxicity, rapid metabolism, and rapid onset/offset of analgesic effect).

Chloroprocaine has an optimal pharmacokinetic profile, and it is promising from the perspective of continuous infusion in the postoperative period. ${ }^{11-13}$ Furthermore, we have recently demonstrated that a continuous intrawound infusion of chloroprocaine can also reduce perilesional inflammation in an animal model. ${ }^{14}$

Finally, nanotechnology meanwhile offers new potential advantages in terms of controlled and sustained release of drugs over several days.

We present data on a chloroprocaine-loaded hydrogel device that might offer revolutionary advantages for postoperative analgesia through its specific pharmacokinetic profile, supported by a continuous hydrogel device administration. In this study, we analyzed both in vitro and in vivo (piglets receiving slow-release chloroprocaine) pharmacokinetic profiles of the chloroprocaine-loaded device.

\section{Materials and methods Materials}

Alginate-based hydrogels were obtained by calcium crosslinking starting from an internal gelation mechanism. ${ }^{15,16}$ Hydrogels were produced from an alginate (alginic acid sodium salt powder, 180947, lot MKBJ0727V; SigmaAldrich Co., St Louis, MO, USA) solution with an initial concentration of $6.67 \%(\mathrm{w} / \mathrm{v})$. For HPLC analysis, highpurity water (CHROMASOLV ${ }^{\circledR}, 34877$; Sigma-Aldrich Co.) was used for the preparation of solutions and suspensions. The drug (chloroprocaine powder, Lot: \#140376; Sintetica Company, Mendrisio, Switzerland), pre-suspended in water and mixed with a diluted solution of alginate $(0.5 \% \mathrm{w} / \mathrm{v})$, was added to the initial solution. The final concentrations were $36 \mathrm{mg} / \mathrm{mL}$ alginate loaded with $75 \mathrm{mg} / \mathrm{mL}$ of chloroprocaine. To prevent bacterial contamination, ethanol-washed alginate was produced by suspending $10 \mathrm{~g}$ alginic acid sodium salt in $50 \mathrm{~mL}$ of absolute ethanol. The procedure was repeated three times. In each step, alginate was suspended in ethanol for 5 minutes, left to sediment for 10 minutes, subsequent to which the supernatant was removed. Following the final wash, the remaining ethanol was allowed to evaporate for 48 hours. All the processes (including preparation of alginate solution) took place under laminar flow. Solutions and water were filtered with $0.22 \mu \mathrm{m}$ filters. The hydrogels were produced within an ad hoc mol $(14 \mathrm{~cm} \times 2 \mathrm{~cm} \times 0.4 \mathrm{~cm})$ for implantation and testing, unless diversely specified.

\section{In vitro chloroprocaine release profile UV analysis}

A chloroprocaine calibration curve was obtained for a physiological-like solution ( $\mathrm{NaMgCa}$ medium, $145 \mathrm{mM}$ $\mathrm{NaCl}, 1.5 \mathrm{mM} \mathrm{MgCl}_{2}$, and $2.5 \mathrm{mM} \mathrm{CaCl}_{2}$ ) measuring solution absorbance with UV-spectroscopy technique (UV/visible Spectrophotometer; Jenway, Staffordshire, UK) in a range of 200-500 nm. NaMgCa medium was developed with the aim of mimicking human plasma concentration considering not only monovalent ions $\left(\mathrm{Na}^{+}\right)$but also divalent ions $\left(\mathrm{Ca}^{2+}\right.$ and $\mathrm{Mg}^{2+}$ ). Medium composition presents a $\mathrm{Na}^{+}$concentration that is isotonic to human plasma and the identical concentration of $\mathrm{Mg}^{2+}$ and $\mathrm{Ca}^{2+}$ in human plasma. From a stock drug solution in $\mathrm{NaMgCa}$ medium with a concentration of $1.5 \mathrm{mg} / \mathrm{mL}$, different solutions in the concentration range of 2.5-120 $\mu \mathrm{g} /$ $\mathrm{mL}$ were prepared by subsequent dilution. All measurements were done in $10 \mathrm{~mm}$ quartz cells (108-QS; Hellma Analytics, Müllheim, Germany). A calibration curve was obtained considering the absorbance peak at $291 \mathrm{~nm}$.

Drug release was performed from hydrogel samples with $15.4 \mathrm{~mm}$ diameter and $3 \mathrm{~mm}$ thickness in $1 \mathrm{~mL}$ of medium. Samples were put in contact with $\mathrm{NaMgCa}$ medium for 15 minutes, 30 minutes, 1 hour, 2 hours, 3 hours, 4 hours, 5 hours, 6 hours, and 24 hours, collecting the medium after each time point and replacing it with fresh medium.

All measurements were calculated in duplicate and in at least two distinct experiments.

\section{HPLC/tandem mass spectrometry (HPLC-MS/MS)} analysis

HPLC-MS/MS (TSQ Quantum Access; Thermo Fisher Scientific, Waltham, MA, USA) analytical method was 
used to quantify chloroprocaine and its inactive metabolite 4-amino-2-chlorobenzoic acid (ACBA) in plasma and acidified methanol, following in vivo and in vitro tests, respectively. In brief, four nanostructured devices containing chloroprocaine and four unfilled nanogel devices (used as a reference) remained in contact with plasma at $37^{\circ} \mathrm{C}$ for 90 minutes, 3 hours, 1 day, and 7 days (four samples) for HPLC-MS/MS analysis.

Mobile phases A (ultrapure water, Millipore Direct-Q ${ }^{\mathrm{TM}}$ system) and $\mathrm{B}$ ( $\mathrm{CH}_{3} \mathrm{OH}, 34860-2.5 \mathrm{~L}-\mathrm{R}$; Lot: \# STBF5815V; Sigma-Aldrich Co.) were both acidified with formic acid (33015-1L; Lot: \# SZBF1540V).

Chloroprocaine chlorohydrate powder (Lot: \#140376; Sintetica Company) and its inactive metabolite (ACBA) were obtained from Sigma Aldrich Co. (217719-5G; Lot: \# $\mathrm{S} 54349 \mathrm{~V})$ and used to prepare the standard solutions. Lidocaine $(20 \mathrm{mg} / \mathrm{mL}$; Bioindustria L.I.M S.p.a, Fresonara, Italy) was used (following dilution) as an internal standard (IS). Stock solutions of chloroprocaine and ACBA were prepared in ultrapure water (chloroprocaine: $1 \mathrm{mg} / \mathrm{mL}$; ACBA: $1 \mathrm{mg} / \mathrm{mL}$ ).

The separation of the two analytes was performed using a Thermo Scientific Accela system, a quaternary pump coupled with an autosampler, and a Kinetex ${ }^{\circledR} 2.6 \mu \mathrm{C} 18100 \AA$ Column $\left(100 \times 4.6 \mathrm{~mm}^{2}\right.$; Phenomenex, Bologna, Italy) with the related guard columns. The columns were also heated and maintained at $40^{\circ} \mathrm{C}$; elution was carried out in the gradient mode, at a flow rate of $0.8 \mathrm{~mL} / \mathrm{min}$, with both the mobile phases $\mathrm{A}$ and B freshly prepared. The injection volume was $2.0 \mu \mathrm{L}$.

A TSQ Quantum Access triple quadrupole mass spectrometer (Thermo Fisher Scientific) with an electrospray ionization probe was used. We set up the analytical system in a multireaction monitoring mode, following the transitions $\mathrm{m} / \mathrm{z} 271 \rightarrow 154,198 ; \mathrm{m} / \mathrm{z} 172 \rightarrow 90,154 ;$ and $\mathrm{m} / \mathrm{z}$ $235 \rightarrow 86.2$ for chloroprocaine, ACBA, and lidocaine, respectively.

Xcalibur 2.07 and LCquan 2.5.6 software from Thermo Fisher Scientific for the HPLC-MS/MS system control, data acquisition, and data analysis were used.

The calibration curves were generated from two different weighted $(1 / \mathrm{x})$ linear regression curves (for chloroprocaine and ACBA quantitation). Analyte peaks were identified through a combination of retention times and the specific multiple reaction monitoring transitions. The corresponding amounts were quantified by normalizing the peak area to the IS (area analyte/area IS), and concentrations were calculated from the respective calibration curves.

The calibration curves were employed to quantitatively evaluate the drug release in plasma and to assess its relative stability in plasma. The hydrogel samples were incubated in direct contact with plasma, at $37^{\circ} \mathrm{C}$, for up to 7 days.

All measurements were calculated in duplicate and in at least two distinct experiments.

\section{Sample preparation for the detection of} chloroprocaine and ACBA analysis for "in vitro tests" The preliminary analyses showed a high concentration of chloroprocaine and its metabolite $(\mathrm{mg} / \mathrm{mL})$ following exposure of the plasma to the device loaded with the drug, as a consequence of its release.

Before proceeding with the analysis, it was necessary to dilute the samples to a ratio of $1: 10,000$ with acidified $\mathrm{CH}_{3} \mathrm{OH}$ $(0.1 \% \mathrm{HCOOH})$ following processing them, according to the standardized procedures reported subsequently.

Calibrators (A-F) were prepared for quantification and quality controls $(\mathrm{QcH}, \mathrm{QcM}$, and $\mathrm{QcL})$ directly in $\mathrm{CH}_{3} \mathrm{OH} /$ $\mathrm{H}^{+}$, at the concentrations specified subsequently, to compare signals from comparable matrices. The linear correlation coefficients $\left(R^{2}\right)$ of the calibration curves obtained from those calibrators were consistently higher than 0.99 for both chloroprocaine and ACBA.

\section{In vivo tests}

The hydrogels were tested in vivo using piglets as the animal model (pigs weighing $20 \mathrm{~kg}$ ).

All animal procedures were performed in accordance with the protocol approved by the Institutional Animal Care and Use Committee (IACUC) - University of Pavia. The animal care and experimental procedures respected local, national, and European Union guidelines for protection of animals used for scientific purposes (Directive 2010/63/EU - revising Directive 86/609/EEC).

Two different types of tests were performed as follows:

1. In each pig, two incisions were made in the abdominal region, one for the control without the hydrogel and one with the loaded hydrogel.

2. Two incisions were made in the same region, and a hydrogel without the drug (control) was placed in one incision and in the other the loaded hydrogel was placed. The incision was performed by simulating a laparotomy $(15 \mathrm{~cm})$, cutting the skin, the abdominal fat, the abdominal muscles, and the peritoneum. Prior to the hydrogel insertion, the peritoneum was sutured. During the surgery, the pigs were anesthetized and all the materials used were sterile. The animals were routinely monitored for general appearance, activity, and healing of the implant sites and were sacrificed after 21 days. No pigs were lost during the study. 
The surgery was performed simulating a laparotomy $(15 \mathrm{~cm})$, cutting the skin, the abdominal fat, the abdominal muscles, and the peritoneum (Figure 1A). The peritoneum was then sutured (Figure 1B), the device was inserted (Figure 1C), and finally all the other tissues were sutured (Figure 1D).

\section{Working solution preparation for the detection of chloroprocaine and ACBA analysis for "in vivo tests"} Plasma samples of pigs with implanted nanostructured hydrogel devices were analyzed to evaluate chloroprocaine release.

Calibrators and the calibration curves in pigs' plasma collected immediately prior to treatment (reference plasma) were prepared as described in the following sections.

Chloroprocaine chlorohydrate powder was weighed and dissolved in water to obtain two distinct stock solutions $\left(\mathrm{M}_{1}\right.$ and $\mathrm{M}_{1 \mathrm{QC}}: 1 \mathrm{mg} / \mathrm{mL}$ ) to be used for the preparation of the six calibrators and three quality control working standard solutions - labeled A, B, C, D, E, F, and QcH, QcM, and QcL, respectively - by a serial dilution procedure.

Analogously, ACBA stock solution ( $1 \mathrm{mg} / \mathrm{mL}$ in $\mathrm{CH}_{3} \mathrm{OH}$ ) and the related working standard solutions for the calibrators and quality control preparations were prepared.
The stock solutions $\mathrm{M}_{1}$ and $\mathrm{M}_{1 \mathrm{QC}}$ (chloroprocaine and ACBA) were stored at $-20^{\circ} \mathrm{C}$ until use, whereas the working standard solutions were prepared on a daily basis.

\section{Preparation of calibrators, quality controls, and plasma samples}

Starting with $90 \mu \mathrm{L}$ of reference plasma and $10 \mu \mathrm{L}$ of each working solution, six calibrators $(\mathrm{A}-\mathrm{F})$ and three quality controls (QcH, QcM, and QcL) were prepared, each containing both chloroprocaine and the inactive metabolite ACBA at the desired concentrations: F $15.65 \mathrm{ng} / \mathrm{mL}$, E $31.25 \mathrm{ng} /$ mL, D $62.5 \mathrm{ng} / \mathrm{mL}$, C $125 \mathrm{ng} / \mathrm{mL}$, B $250 \mathrm{ng} / \mathrm{mL}$, A $500 \mathrm{ng} /$ mL; QcH 400 ng/mL, QcM 200 ng/mL, and QcL 20 ng/mL.

The IS (lidocaine) and methanol were added to each sample ( $100 \mu \mathrm{L}$ of pig's plasma) to promote protein precipitation. After mixing via vortex and centrifuge separation, the supernatant was recovered and dried under a weak stream of nitrogen. Next, the precipitate was suspended in acidified methanol and directly injected into the column.

Both the corresponding calibration curves of chloroprocaine and ACBA demonstrated an excellent linearity in the considered concentration range (15.65-500 ng/mL), characterized by a correlation coefficient $\left(R^{2}\right)$ consistently higher than 0.99 .

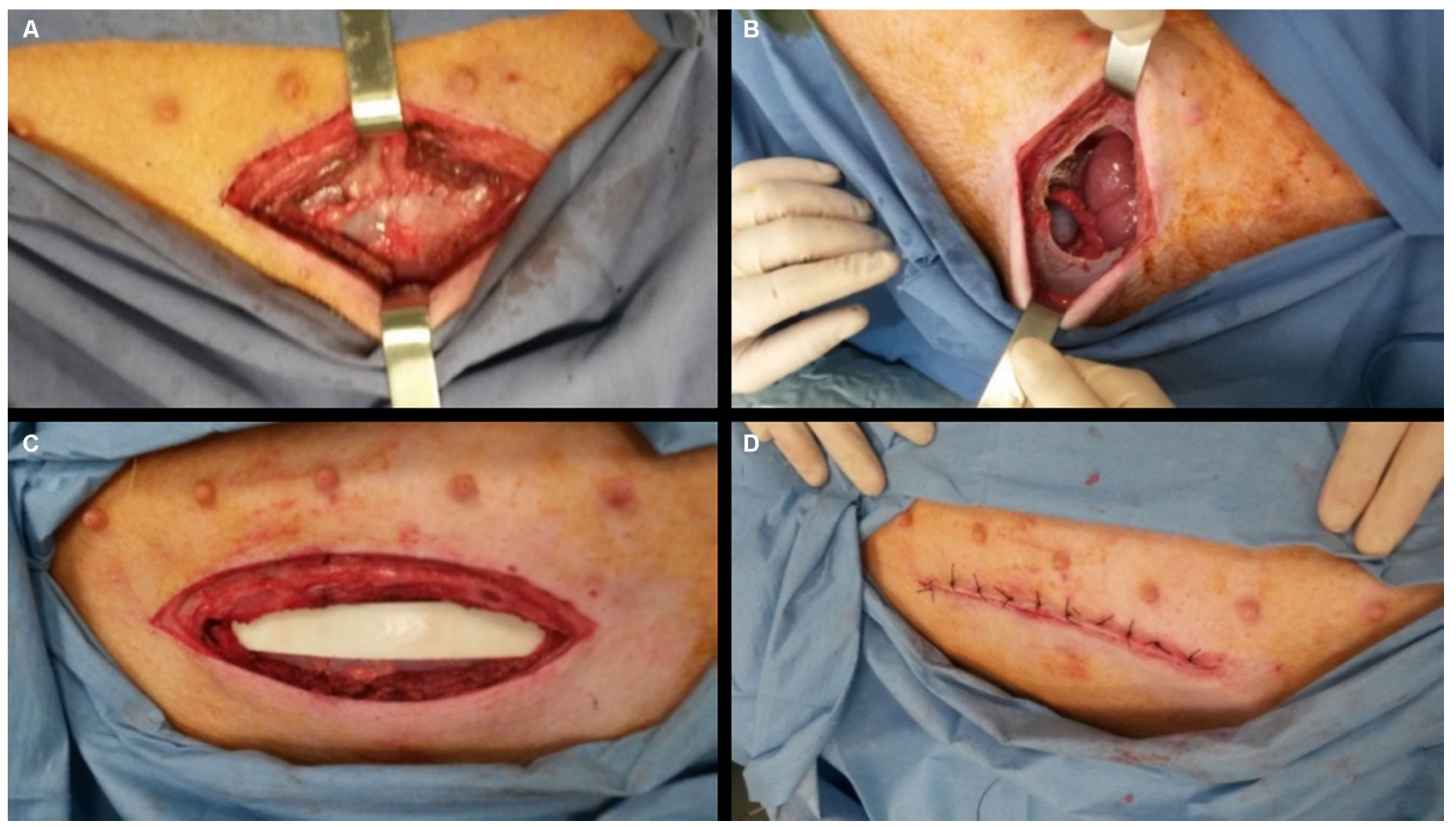

Figure I Different steps of surgery.

Notes: (A) Incision of skin, abdominal fat, abdominal muscles, and peritoneum. (B) Suture of peritoneum. (C) Device insertion. (D) Suture of all other tissues. 


\section{Acceptance criteria of an analytical run}

As reported in the "Guideline on bioanalytical method validation" (EMA/CHMP/EWP/192217/2009 Rev.1 Corr.*), the back calculated concentrations of the calibration standards should be within $\pm 15 \%$ of the nominal value, with the exception of the lower limit of quantification (LLOQ), for which it should be within $\pm 20 \%$. At least $75 \%$ of the calibration standards ( 1 over 6$)$, with a minimum of six, are required to fulfill this criterion. If one of the calibration standards does not meet these criteria, this calibration standard should be rejected and the calibration curve without this calibration standard should be re-evaluated, and regression analysis should be performed. The accuracy values of the Qc samples should be within $\pm 15 \%$ of the nominal values. At minimum $67 \%$ of the Qc samples and $50 \%$ at each concentration level should comply with this criterion. If these criteria are not fulfilled, the analytical run should be rejected, and the study samples re-extracted and analyzed.

\section{Results}

In vitro evaluation of chloroprocaine-loaded hydrogel release UV spectroscopy and HPLC-MS/MS analytical method was performed for in vitro evaluation of the chloroprocaine release from the nanostructured hydrogel device.

\section{UV analysis of chloroprocaine release}

For initial quantitative evaluation of the drug in release medium, UV spectroscopy was employed to correlate the concentration of the drug with the absorbance. Chloroprocaine spectra at different concentrations in medium are displayed in Figure 2. For concentrations above $50 \mu \mathrm{g} / \mathrm{mL}$, absorbance resulted in out-of-scale peaks. The spectra present characteristic peaks of different absorbance intensities.

The chloroprocaine-loaded hydrogel sample demonstrated an initial release rate of $22.5 \mathrm{mg} / \mathrm{h}$, and in the following hours, a constant rate of $\sim 8.0 \mathrm{mg} / \mathrm{h}$. The release decreased after 5 hours and was prolonged until 24 hours, at which point the detection limit was reached (Figure 2B).

\section{Evaluation of chloroprocaine and ACBA metabolite release by HPLC-MS/MS}

For HPLC-MS/MS detection, four nanostructured devices containing chloroprocaine and four unfilled nanogel devices (used as reference) remained in contact with plasma at $37^{\circ} \mathrm{C}$ for 90 minutes, 3 hours, 1 day, and 7 days (four samples). The plasma samples were analyzed via HPLC-MS/MS to assess chloroprocaine and $\mathrm{ABCA}$ concentrations.

The obtained concentrations for chloroprocaine and its ACBA metabolite were $0.522-5.436$ and $2.84-8.348 \mathrm{mg} / \mathrm{mL}$, respectively (Figure 3 ).

The analyses produced a peak of maximum plasma concentration $\left(\mathrm{C}_{\max }\right)$ of chloroprocaine after 1 day of exposure to the device $(5.436 \mathrm{mg} / \mathrm{mL})$ and a minimum $\left(\mathrm{C}_{\min }\right)$ after 7 days $(0.522 \mathrm{mg} / \mathrm{mL})$.

Otherwise, ACBA reached $\mathrm{C}_{\max }$ after 7 days $(8.348 \mathrm{mg} /$ $\mathrm{mL})$.
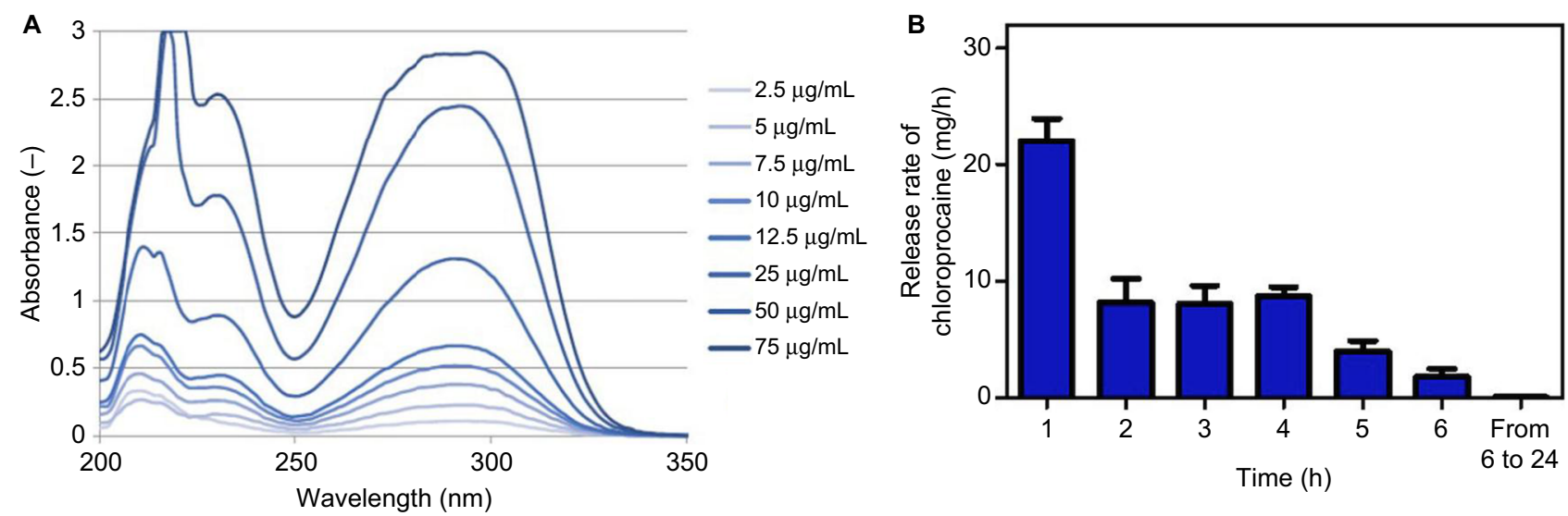

Figure 2 (A) Chloroprocaine UV spectra at different concentrations in $\mathrm{NaMgCa}$ medium. (B) Chloroprocaine release from hydrogel device measured at different time points.

Notes: A standard curve $\left(y=62,194 \mathrm{x}+0.0837 ; R^{2}=0.9958\right)$ was obtained from the absorbance of the band at $291 \mathrm{~nm}$ for chloroprocaine, as it was the only one clearly detectable for the range of concentrations analyzed $(2.5-75 \mu \mathrm{g} / \mathrm{mL})$. It was not possible to apply UV spectroscopy to evaluate concentrations below $2.5 \mu \mathrm{g} / \mathrm{mL}$, the LLOQ. Abbreviations: h, hours; LLOQ, lower limit of quantification. 


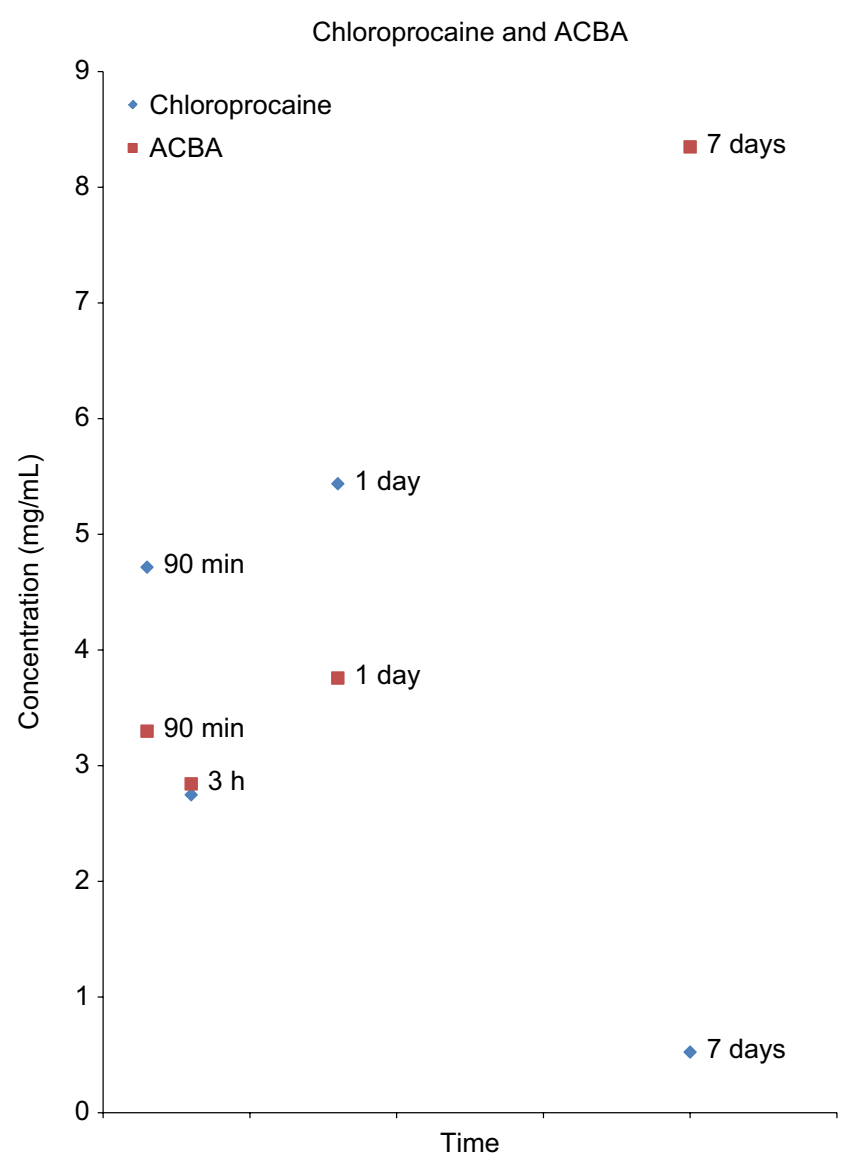

Figure 3 In vitro detections through HPLC-MS/MS of the concentrations of chloroprocaine and ACBA metabolite released from drug-loaded hydrogel after being in contact with plasma at $37^{\circ} \mathrm{C}$ for 90 minutes, 3 hours, I day, and 7 days (four samples), respectively.

Abbreviations: ACBA, 4-amino-2-chlorobenzoic acid; HPLC-MS/MS, HPLCtandem mass spectrometry; $h$, hours; min, minutes.

Chloroprocaine plasma concentrations were undetectable in all the reference samples; in contrast, traces of ACBA were found in all the samples.

\section{In vivo evaluation of the chloroprocaine- loaded hydrogel release}

For each of the four piglets (named Gelly 2, Gelly 3, Gelly 4, and Gelly 5), 11 plasma samples were analyzed and identified as basal, $\mathrm{T}_{0}, \mathrm{~T}_{3 \mathrm{~h}}, \mathrm{~T}_{6 \mathrm{~h}}, \mathrm{~T}_{12 \mathrm{~h}}, \mathrm{~T}_{24 \mathrm{~h}}, \mathrm{~T}_{48 \mathrm{~h}}, \mathrm{~T}_{72 \mathrm{~h}}, \mathrm{~T}_{96 \mathrm{~h}}, \mathrm{~T}_{102 \mathrm{~h}}$, and $\mathrm{T}_{108 \mathrm{~h}}$, according to the length of exposure (measured in hours) to the device. The basal sample was never in contact with the device (Figure 4), whereas the $\mathrm{T}_{0}$ sample remained in contact with the device for less than 3 hours.

As expected, signals of ACBA, lidocaine, and chloroprocaine were absent in the plasma of the untreated piglets.

No quantifiable amount of chloroprocaine was identified in any of the plasma samples.
The detected ACBA plasma concentrations are reported in Table 1. The LLOQ was $15 \mathrm{ng} / \mathrm{mL}$.

Plasma concentrations were detectable ( $\geq$ LLOQ), primarily between $\mathrm{T}_{0}$ and $\mathrm{T}_{12 \mathrm{~h}}$.

The $\mathrm{T}_{0}$ plasma concentration of Gelly 2 (although revalued several times) was an outlier (3,209.42 ng/mL).

The peak of maximum ACBA plasma concentration $\left(\mathrm{C}_{\max }\right)$ was observed 3 hours following the device application (Table 1).

Although the chloroprocaine plasma concentrations were consistently below the LLOQ, a well-defined peak in the chromatograms of each animal (between $\mathrm{T}_{3 \mathrm{~h}}$ and $\mathrm{T}_{6 \mathrm{~h}}$ ) was detected, albeit at a different retention time from that expected.

In order to identify their well-defined retention times, some working solutions of ABCA, lidocaine, and chloroprocaine were at first added to a piglet-free drug plasma and analyzed; the related chromatographic peaks were obtained at 2.82, 2.73, and 1.90 minutes, respectively (Figure 5).

On the contrary, unexpected peaks at 2.58 minutes (differing from 1.90 minutes) were observed in the chloroprocaine mass channel during the chromatographic runs of Gelly 2, Gelly 3, Gelly 4, and Gelly 5, and they were observed between $\mathrm{T}_{3 \mathrm{~h}}$ and $\mathrm{T}_{6 \mathrm{~h}}$.

\section{Discussion}

LAs are useful in treating acute POP, ${ }^{17}$ although severe side effects are related to their systemic usage (lidocaine and bupivacaine). ${ }^{18,19}$ Less toxic LAs (eg, chloroprocaine) have been developed, but present short half-lives. ${ }^{20}$ To prolong drug effectiveness and minimize drawbacks, LA drug delivery systems have been developed. ${ }^{1}$

Currently, hydrogels are being widely exploited in biomedical applications as drug delivery systems. An innovative aspect of the present work regards the use of alginate, commonly employed to enhance biocompatibility of PLGA microspheres or as coating to tailor drug release, ${ }^{21-23}$ as a principal material for hydrogel device development.

Alginate is an anionic naturally occurring biopolymer, typically extracted from brown algae, that has many possible applications in different biomedical applications. The favorable properties of alginate, including biocompatibility and ease of gelation, enable its use as a matrix for the entrapment and delivery of proteins, drugs, cells, wound healing, and tissue engineering. ${ }^{24,25}$

The usage of an anionic polymer could also have the advantage of creating electrostatic interactions between polymer and drug, which may reduce drug diffusion coefficients, prolonging the release time. Although alginate is inherently non-degradable in mammals as they lack the 


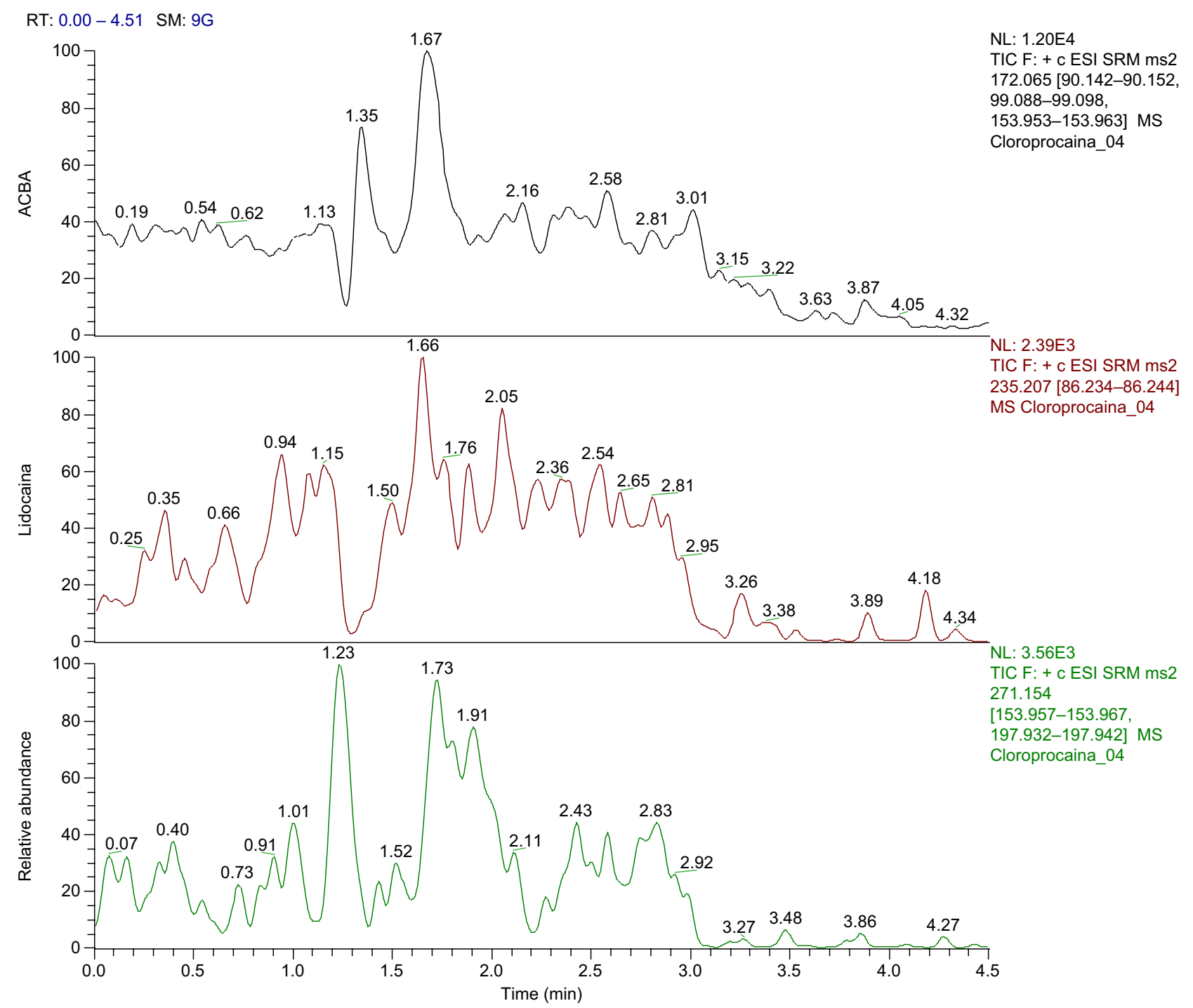

Figure 4 Chromatograms of a piglet plasma sample, prior to application of the nanostructured hydrogel device (basal sample).

Abbreviations: ACBA, 4-amino-2-chlorobenzoic acid; + c ESI, a positive, centroid scan, acquired from ions generated by an electrospray ion source (ionization mode); min, minutes; F, scan filter; ms2, tandem mass spectrometry; MS, mass; NL, normalize; RT, retention time; SRM, selected reaction monitoring; TIC, total ion current.

Table I ACBA plasma concentrations in four piglets following application of the nanostructured drug-loaded hydrogel

\begin{tabular}{|c|c|c|c|c|c|c|c|c|c|c|c|}
\hline \multirow{2}{*}{$\begin{array}{l}\text { Sample } \\
\text { name }\end{array}$} & \multicolumn{11}{|c|}{ ACBA $(n g / m L)$} \\
\hline & Basal & $\mathbf{T}_{0}$ & 3 hours & 6 hours & I 2 hours & 24 hours & 48 hours & 72 hours & 96 hours & 102 hours & 108 hours \\
\hline Gelly 2 & N.C & $3,209.4$ & 477.01 & 228.22 & 69.16 & N.C & N.C & N.C & 15.53 & N.C & N.C \\
\hline Gelly 3 & N.C & 109.25 & 524.14 & 216.31 & 55.50 & N.C & 15.09 & N.C & 19.53 & N.C & N.C \\
\hline Gelly 4 & N.C & 19.67 & 470.44 & 378.73 & 83.00 & N.C & N.C & N.C & N.C & N.C & N.C \\
\hline Gelly 5 & N.C & 393.53 & 1330.4 & 313.69 & 444.38 & N.C & N.C & N.C & N.C & N.C & N.C \\
\hline
\end{tabular}

Abbreviations: ACBA, 4-amino-2-chlorobenzoic acid; N.C, not calculable.

enzyme (ie, alginase) which can cleave the polymer chains, it has been reported that the degradation of alginate gels can be controlled by the release of the divalent ions involved in the gel cross-linking into the surrounding media via exchange reactions with sodium ions. ${ }^{25}$ Furthermore, as already evi- denced by other studies, the partial oxidation of alginate hydrogels can produce an easy degradation in vivo, ${ }^{26}$ which constitutes further evidence supporting the clinical utilization of alginate as a device that will not need to be removed after its implantation. All these features, including its anatomical 


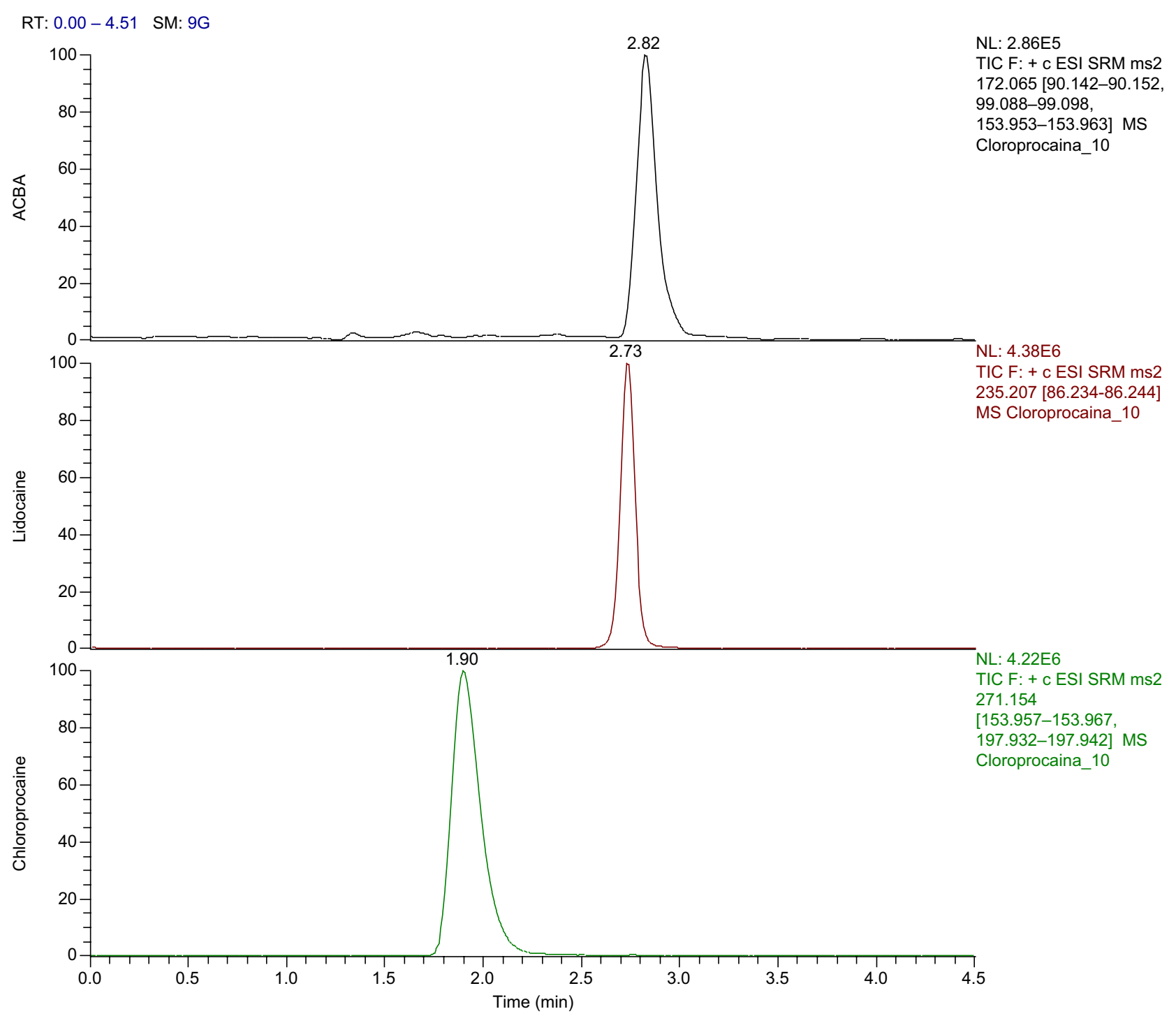

Figure 5 Chromatograms of a piglet's plasma sample, added with chloroprocaine, ACBA, and lidocaine, as an internal standard.

Abbreviations: ACBA, 4-amino-2-chlorobenzoic acid; + c ESI, a positive, centroid scan, acquired from ions generated by an electrospray ion source (ionization mode); min, minutes.

compatibility with soft tissues, make alginate suitable for the controlled release of LAs as an alternative to the use of synthetic biodegradable polyesters.

Through an UV and an HPLC-MS/MS system, we analyzed in vitro and in vivo release of an experimental chloroprocaine-loaded hydrogel sample. First, in vitro results demonstrated an initial release rate of $22.5 \mathrm{mg} / \mathrm{h}$ and in subsequent hours, a constant rate of $\sim 8.0 \mathrm{mg} / \mathrm{h}$. The release decreased after 5 hours and was prolonged until 24 hours. Further analysis associated the decrease in chloroprocaine concentration with an increase in ACBA concentration, indicating additionally an in vitro drug degradation.

From an in vivo analysis, we found that at the specified sampling time, LA's concentration was never detectable. Conversely,
ACBA was quantified in almost all plasma samples, with a peak of $\mathrm{C}_{\text {max }}$ observed 3 hours following the device application.

The $\mathrm{T}_{0}$ concentration value of Gelly 2 (although revalued several times) was found not to align with that of the others. We assume that blood was incorrectly collected immediately following the nanodevice hydrogel device application rather than prior to the application.

Moreover, the presence of a molecule similar to chloroprocaine was detected through molecular weight and the same fragmentation pattern, although differed structurally. This may have resulted from specific interactions with the stationary phase of the analytical column and the mobile phases of another chloroprocaine metabolite undergoing an in-source fragmentation producing chloroprocaine. ${ }^{27-29}$ 
In vitro, the $\mathrm{ACBA}$ metabolite reached the $\mathrm{C}_{\max }$ after 7 days, whereas chloroprocaine obtained a $\mathrm{C}_{\max }$ following only 1 day of exposure to the device, and a minimum $\left(\mathrm{C}_{\min }\right)$ after 7 days. These results suggest that the drug degraded slowly over time. As was the case in the analysis of reference samples, quantifiable traces of the metabolite emerged, and it was assumed that the device released some material, initially present on the surface of contact with the plasma.

The presented data suggest that the hydrogel device maintains the structural characteristics of drugs. Therefore, the initial rapid drug release may be effective in treating POP in the initial postoperative hours, while a reduced rate of release may be effective to prevent chronic pain in the subsequent period.

In the near future, we intend to repeat the analyses on the reference samples, starting from devices for which no release is expected, placed only in contact with serum and then water. Finally, we will validate the pharmacokinetic results by substituting the procaine for the chloroprocaine. Doing so will highlight any differences between the two drugs in terms of release and degradation processes.

By following a precision medicine approach, drug loading will be modulated depending on the type of pain to be managed. We are hopeful that such translational research will soon result in the clinical utilization of the hydrogel device.

\section{Limitations of the study}

This study reports qualitative results of a device implanted in piglets, to evaluate chloroprocaine release, for potential future management of human POP. Piglets, as larger animals, are considered the most similar pharmacokinetic model to human being in terms of experimental response, ${ }^{30}$ although the cost of piglets compared to rats necessitates a smaller sample size. This limitation, as well as the absence of previous literature data regarding analog in vitro and in vivo results, does not allow us to perform a power statistical analysis or to support any power calculation. Accordingly, the present study is to be considered a pilot, and our expectation is that future, larger investigations will allow us to move closer to the ultimate goal of human application in the clinical setting.

\section{Conclusion}

The experimental chloroprocaine-loaded hydrogel sample was developed to produce a new effective analgesic method, which will contribute to improving POP relief.

\section{Acknowledgments}

The study has been funded by a grant by the Italian Health Ministry ("New nanotechnology and biomedical approaches to improve postoperative pain treatment reducing risks related to opioids" - GR-2010-2318370; Principal Investigator Massimo Allegri, MD) and a grant by Foundation "Banca del Monte di Lombardia" ("La Nanotecnologia applicata al Trattamento del dolore"; Principal Investigator Lorenzo Cobianchi, MD, USA). The authors would like to thank Paola Petrini from Politecnico di Milano for providing the hydrogels used in this work, Livia Visai, Università di Pavia, for the in vitro tests, and Sintetica ${ }^{\circledR}$ for providing chloroprocaine for this study.

\section{Disclosure}

The authors report no conflicts of interest in this work.

\section{References}

1. Zorzetto L, Brambilla P, Marcello E, et al. From micro- to nanostructured implantable device for local anesthetic delivery. Int J Nanomedicine. 2016;11:2695-2709.

2. Kuhnert BR, Kuhnert PM, Philipson EH, Syracuse CD, Kaine $\mathrm{CJ}$, Yun CH. The half-life of 2-chloroprocaine. Anesth Analg. 1986;65(3):273-278.

3. Taraballi F, Minardi S, Corradetti B, et al. Potential avoidance of adverse analgesic effects using a biologically "smart" hydrogel capable of controlled bupivacaine release. J Pharm Sci. 2014;103(11):3724-3732.

4. Grillo R, de Melo NF, de Araújo DR, et al. Polymeric alginate nanoparticles containing the local anesthetic bupivacaine. J Drug Target. 2010;18(9):688-699.

5. Li J, Mooney DJ. Designing hydrogels for controlled drug delivery. Nat Rev Mater. 2016;1(12):16071.

6. Liu Z1, Jiao Y, Wang Y, Zhou C, Zhang Z. Polysaccharides-based nanoparticles as drug delivery systems. Adv Drug Deliv Rev. 2008;60(15):1650-1662.

7. Gordon DB, de Leon-Casasola OA, Wu CL, Sluka KA, Brennan TJ, Chou R. Research gaps in practice guidelines for acute postoperative pain management in adults: findings from a review of the evidence for an american pain society clinical practice guideline. J Pain. 2016;17(2):158-166.

8. Golembiewski J, Dasta J. Evolving role of local anesthetics in managing postsurgical analgesia. Clin Ther. 2015;37(6):1354-1371.

9. Rivat C, Bollag L, Richebé P. Mechanisms of regional anaesthesia protection against hyperalgesia and pain chronicization. Curr Opin Anaesthesiol. 2013;26(5):621-625.

10. Dhanapal B, Sistla SC, Badhe AS, Ali SM, Ravichandran NT, Galidevara I. Effectiveness of continuous wound infusion of local anesthetics after abdominal surgeries. J Surg Res. 2017;212:94-100.

11. Gebhardt V, Kiefer K, Bussen D, Weiss C, Schmittner MD. Retrospective analysis of mepivacaine, prilocaine and chloroprocaine for low-dose spinal anaesthesia in outpatient perianal procedures. Int J Colorectal Dis. 2018;33(10):1469-1477.

12. Teunkens A, Vermeulen K, van Gerven E, Fieuws S, van de Velde M, Rex S. Comparison of 2-chloroprocaine, bupivacaine, and lidocaine for spinal anesthesia in patients undergoing knee arthroscopy in an outpatient setting: a double-blind randomized controlled trial. Reg Anesth Pain Med. 2016;41(5):576-583.

13. Förster JG, Rosenberg PH, Harilainen A, Sandelin J, Pitkänen MT. Chloroprocaine $40 \mathrm{mg}$ produces shorter spinal block than articaine 40 $\mathrm{mg}$ in day-case knee arthroscopy patients. Acta Anaesthesiol Scand. 2013;57(7):911-919.

14. Allegri M, Bugada D, de Gregori M, et al. Continuous wound infusion with chloroprocaine in a pig model of surgical lesion: drug absorption and effects on inflammatory response. J Pain Res. 2017;10:2515-2524. 
15. Moreira HR, Munarin F, Gentilini R, et al. Injectable pectin hydrogels produced by internal gelation: $\mathrm{pH}$ dependence of gelling and rheological properties. Carbohydr Polym. 2014;103:339-347.

16. Gentilini R, Munarin F, Bloise N, et al. Polysaccharide-based hydrogels with tunable composition as 3D cell culture systems. Int JArtif Organs. 2018;41(4):213-222.

17. Andreae MH, Andreae DA. Local anaesthetics and regional anaesthesia for preventing chronic pain after surgery. Cochrane Database Syst Rev. 2012;10:CD007105.

18. Gitman M, Barrington MJ. Local anesthetic systemic toxicity: a review of recent case reports and registries. Reg Anesth Pain Med. 2018;43(2):124-130.

19. Allegri M, Bugada D, Grossi P, et al. An incidence analysis from a prospective clinical survey. Minerva Anestesiol. 2016;82(4):392-402.

20. Kuhnert BR, Kuhnert PM, Philipson EH, Syracuse CD, Kaine CJ, Chang-Hyon Y. The Half-Life of 2-Chloroprocaine. Anesth Analg. 1998;65:273-278.

21. Liu DZ, Sheu MT, Chen CH, Yang YR, Ho HO. Release characteristics of lidocaine from local implant of polyanionic and polycationic hydrogels. J Control Release. 2007;118(3):333-339.

22. Zhang H, Lu Y, Zhang G, Gao S, Sun D, Zhong Y. Bupivacaine-loaded biodegradable poly(lactic-co-glycolic) acid microspheres I. Optimization of the drug incorporation into the polymer matrix and modelling of drug release. Int J Pharm. 2008;351(1-2):244-249.
23. Cong Z, Shi Y, Wang Y, et al. A novel controlled drug delivery system based on alginate hydrogel/chitosan micelle composites. Int J Biol Macromol. 2018;107(Pt A):855-864.

24. Sriamornsak P, Thirawong N, Korkerd K. Swelling, erosion and release behavior of alginate-based matrix tablets. Eur J Pharm Biopharm. 2007;66(3):435-450.

25. Lee KY, Mooney DJ. Alginate: properties and biomedical applications. Prog Polym Sci. 2012;37(1):106-126.

26. Reakasame S, Boccaccini AR. Oxidized alginate-based hydrogels for tissue engineering applications: A Review. Biomacromolecules. 2018;19(1):3-21

27. Xu YF, Lu W, Rabinowitz JD, Joshua D. Avoiding misannotation of in-source fragmentation products as cellular metabolites in liquid chromatography-mass spectrometry-based metabolomics. Anal Chem. 2015;87(4):2273-2281.

28. Demarque DP, Crotti AE, Vessecchi R, Lopes JL, Lopes NP. Fragmentation reactions using electrospray ionization mass spectrometry: an important tool for the structural elucidation and characterization of synthetic and natural products. Nat Prod Rep. 2016;33(3):432-455.

29. Yan Z, Caldwell GW, Jones WJ, Masucci JA. Cone voltage induced insource dissociation of glucuronides in electrospray and implications in biological analyses. Rapid Commun Mass Spectrom. 2003;17(13):1433-1442.

30. Gigliuto $\mathrm{C}$, de Gregori M, Malafoglia V, et al. Pain assessment in animal models: do we need further studies? J Pain Res. 2014;7:227-236.
Journal of Pain Research

\section{Publish your work in this journal}

The Journal of Pain Research is an international, peer reviewed, open access, online journal that welcomes laboratory and clinical findings in the fields of pain research and the prevention and management of pain. Original research, reviews, symposium reports, hypothesis formation and commentaries are all considered for publication.

\section{Dovepress}

The manuscript management system is completely online and includes a very quick and fair peer-review system, which is all easy to use. Visit http://www.dovepress.com/testimonials.php to read real quotes from published authors. 